\title{
ascrègep
}

\section{Politique de la recherche}

92.06.16.06 - amendée 07.06.20.09 - amendée 09.02.25.12 - amendée 2012.06.13.13

\section{Préambule}

La Loi sur les Collèges d'enseignement général et professionnel précise, à l'article 6.01.b, que les collèges peuvent effectuer des études et effectuer des recherches et soutenir les membres du personnel qui y participent. Pour sa part, le Cégep de Sherbrooke accorde une place importante à la recherche en l'intégrant à ses orientations stratégiques et à ses pratiques notamment en ce qui a trait à l'intégrité en recherche. Il endosse en ce sens l'Énoncé de Politique des Trois Conseils ainsi que le Cadre de référence des trois organismes sur la conduite responsable de la recherche (le Cadre).

La Polique de la recherche doit être considérée en lien étroit avec la Politique sur la conduite responsable de la recherche, la Politique sur les conflits d'intérêts en recherche et la Politique sur l'éthique de la recherche avec des êtres humains.

\section{Champ d'application et cadre juridique}

La présente politique s'applique à tous les types de recherches réalisées par les membres du personnel dans le cadre de leurs activités professionnelles liées au Cégep ou par les étudiantes et étudiants associés à ces activités. Toute activité de recherche doit se faire dans le respect des lois, règles, normes et politiques suivantes le cas échéant:

- la Loi sur les Collèges d'enseignement général et professionnel

- la Charte des droits et libertés de la personne

- la Charte canadienne des droits et libertés

- le Code civil du Québec

- la Loi sur l'accès aux documents des organismes publics et sur la protection des renseignements personnels

- la Loi sur les archives

- le Code des professions du Québec

- la Loi sur les brevets

- la Loi sur les droits d'auteur

- l'Énoncé de politique des trois conseils

- les normes et directives du Conseil canadien de protection des animaux

- la Loi canadienne sur l'évaluation environnementale

- les licences de recherche requises sur le terrain

- les Lignes directrices de biosécurité en laboratoire

- le programme des marchandises contrôlées.

\section{Responsable de l'application}

La direction générale est responsable de l'application de la présente politique. 


\section{Objectifs de la politique}

La présente politique vise principalement à :

- Déterminer le cadre d'exercice, le soutien institutionnel et le partage des responsabilités dans les activités de recherche.

- S'assurer que les principales dispositions législatives québécoises et canadiennes qui balisent la recherche au Cégep sont respectées.

- Favoriser l'intégration de la recherche dans la pratique professionnelle des personnels.

\section{Article 1 - Définitions Chercheur ou chercheuse}

Tout membre du personnel enseignant ou professionel, tout étudiant ou toute étudiante, de même que tout collaborateur ou collabortrice impliqués dans la conduite de projets de recherche.

\section{Développement}

Dans ce contexte, une activité orientée vers l'implantation du changement par la conception et l'élaboration de produits, de techniques ou de services.

\section{Recherche}

Toute activité scientifique contribuant à l'avancement des connaissances en ayant recours à une méthodologie spécifique et explicite.

\section{Recherche-développement en éducation}

Processus utilisant des résultats de recherche pertinents aux fins de conception, de développement et de mise au point des produits éducatifs, des approches pédagogiques et des programmes d'études.

\section{Recherche technologique}

Activités de recherche appliquée orientées vers l'avancement ou l'innovation technologique.

\section{Article 2 - Types de recherche}

Trois types de recherches sont réalisés au Cégep :

- la recherche en éducation qui permet l'avancement des connaissances notamment en enseignement, en didactique et sur l'apprentissage;
- la recherche disciplinaire qui est reliée à un champ de savoir et dont les résultats obtenus contribuent à l'avancement de la discipline en question;

- la recherche technologique dont les activités visent l'application et le transfert de connaissances scientifiques et technologiques au marché du travail.

Note : L'élaboration de matériel didactique sous toutes ses formes, la fabrication d'outils, la collecte de données, les inventaires, les relances, l'administration de tests et de sondage, les activités d'implantation et d'évaluation, les études de clientèles, de pertinence et de faisabilité et les autres opérations pratiquées régulièrement ou occasionnellement au Cégep sans faire partie intégrante d'un projet de recherche, ne sont pas considérés comme des activités de recherche, mais comme des activités connexes à la recherche.

\section{Article 3 - Cadre d'exercice de la recherche}

3.1 La recherche au Cégep se réalise principalement à l'intérieur des cadres d'exercice suivants : la recherche subventionnée par un organisme reconnu, la recherche financée par le Cégep ou la recherche en vue de l'obtention d'un diplôme d'études supérieures.

3.2 Une recherche est subventionnée, lorsque des ententes financières entre les parties - le chercheur ou la chercheuse, l'organisme subventionnaire et le Cégep - permettent la réalisation de la recherche en tout ou en partie. Chaque organisme définit des règles pour l'utilisation des sommes versées et pour la réalisation de la recherche.

3.3 Le Cégep peut préciser des besoins particuliers de recherche et conclure des ententes avec des membres du personnel pour la mise en œuvre de projets.

3.4 Le Cégep peut déterminer et proposer des thèmes de recherche estimés prioritaires pour son développement. 
Article 4 - Principes généraux

4.1 Le Cégep reconnaît que la recherche apporte une contribution essentielle à l'accomplissement de sa mission.

4.2 Le Cégep encourage des activités de recherche contribuant à l'atteinte des objectifs institutionnels.

4.3 Le Cégep crée un climat favorable à l'élaboration et à la réalisation d'activités de recherche, au développement des compétences dans ce champ d'activités et à la qualité des résultats des travaux en ce domaine.

\section{Article 5 - Engagement des chercheurs}

5.1 Les personnes participant à des activités de recherche s'engagent à se conformer aux politiques du Cégep en matière de recherche et aux ententes contractées avec le Cégep et avec les organismes subventionnaires.

5.2 Lors de la formulation d'une entente de financement de projet de recherche, la personne requérante s'engage à assurer l'exécution destravauxprojetés conformémentauxconditions prévues au projet ou au contrat de recherche sous peine de devoir rembourser les sommes dépensées.

5.3 Le Cégep prend les mesures appropriées auprès des chercheurs et chercheuses qui ne respecteraient pas leurs obligations.

5.4 Les personnes qui réalisent des activités de recherche (les chercheurs et les chercheuses) font toujours partie de la communauté collégiale et, à ce titre, sont incitées à participer activement à la vie du Cégep.

\section{Article 6 - Préparation d'une proposition de recherche}

6.1 Lors de la préparation d'une proposition de recherche, la personne requérante voit à l'élaboration de la demande et à l'établissement du budget selon les règles de l'organisme ou du programme sollicité. Elle peut obtenir le soutien-conseil des instances appropriées au type de recherche concernée.
6.2 Avant le dépôt d'une proposition de recherche, la personne requérante doit obtenir l'approbation du Cégep.

6.3 Lors de la préparation d'une proposition de recherche soumise conjointement par plusieurs établissements, la personne requérante veille à établir et à faire connaître au préalable, aux fins d'approbation par chacune des parties, les conditions et les modalités d'engagement et de participation de chacun d'entre eux.

6.4 Lors de la préparation d'une proposition de recherche en éducation et de la réalisation de celle-ci, la personne requérante informe son département ou le service concerné de son projet de recherche, du déroulement et la progression de celui-ci, de même que des résultats qui en ressortent.

6.5 Le Cégep se réserve le droit de refuser toute proposition jugée incompatible avec ses règlements, ses politiques ou ses intérêts, excédant sa capacité organisationnelle ou impropre quant aux exigences de base d'un projet de recherche ou aux exigences de l'organisme subventionnaire visé ou excédant le cadre de la présente politique.

\section{Article 7 - Mesures favorisant la recherche}

7.1 Sous réserve de l'approbation de la supérieure ou du supérieur immédiat, le Cégep assure le dégagement des membres du personnel pour se consacrer à des tâches de recherche chaque fois que le permettent les ressources financières obtenues pour la réalisation des projets de recherche.

7.2 Les départements d'enseignement et les services du Cégep collaborent aux activités de recherche dans leur champ de responsabilité respectif.

7.3 Le Cégep favorise la participation d'étudiantes et d'étudiants à des équipes de recherche dans le cas de projets susceptibles de contribuer à leur formation et de les initier à la recherche. 
7.4 Le Cégep facilite, dans la mesure de ses moyens, la diffusion des résultats de recherche par la participation des auteurs et des auteures de projets de recherche à différents événements publics relatifs à leur champ de recherche.

7.5 Le Cégep, dans le cadre des budgets disponibles aux fins du perfectionnement des membres du personnel, se préoccupe d'offrir des activités de formation à l'intention des personnes impliquées dans des activités que cette implication soit de longue date, récente ou même simplement éventuelle.

7.6 Le Cégep se préoccupe de l'émergence de nouveaux chercheurs et de nouvelles chercheuses en suscitant des candidatures à l'occasion des sessions de perfectionnement, de la formation d'équipes de recherche ou d'échanges avec des personnes déjà engagées dans la recherche.

7.7 Dans le cadre des recherches subventionnées, le Cégep se préoccupe d'offrir un soutien et un encadrement sur mesure aux nouveaux chercheurs et aux nouvelles chercheuses dans la mesure des ressources disponibles.

7.8 Le Cégep confie au Service de soutien à l'enseignement et à la recherche des responsabilités déterminées relativement aux activités de recherche pédagogique ou disciplinaire.

7.9 Le Cégep peut confier au Centre de productique intégrée du Québec inc. des mandats de gestion de projets de recherche technologique.

7.10 Le Cégep peut désigner, selon les circonstances, d'autres organismes pour gérer des projets de recherche.

7.11 Le Service de soutien à l'enseignement et à la recherche s'engage à :

- diffuser les programmes d'aide à la recherche; - fournir de l'aide pour la préparation des propositions de recherche et pour leur acheminement auprès des organismes concernés, sous réserve de l'article 6.2;
- sensibiliser le nouveau personnel à cette perspective de développement professionnel et aux orientations de la recherche préconisées par le Cégep;

- fournir l'encadrement des activités de recherche relevant de sa responsabilité.

\section{Article 8 - Recherche supposant la participation de sujets humains}

8.1 Tous les projets supposant la participation de sujets humains doivent être soumis au Comité d'éthique de la recherche du Cégep (CÉR) lorsque requis et recevoir son approbation avant leur exécution suivant les procédures prévues à la Politique sur l'éthique de la recherche avec des êtres humains.

8.2 En cas de besoin, le Cégep pourra déléguer sa responsabilité d'évaluer la dimension éthique des projets de recherche au Comité d'éthique de la recherche (CÉR) de l'Université de Sherbrooke comme prévu dans les ententes à cet effet. II conservera néanmoins la responsabilité éthique et légale de la recherche qui y est réalisée.

\section{Article 9 - Diffusion de la politique}

La direction générale s'assure de la diffusion de la présente politique auprès de la communauté collégiale et s'assure de son application.

\section{Article 10 - Mise à jour de la politique} La direction générale a la responsabilité de réviser périodiquement et en fonction de l'évolution du cadre juridique et social la présente politique. 